Article

\title{
Functional and Promoter Analysis of ChiIV3, a Chitinase of Pepper Plant, in Response to Phytophthora capsici Infection
}

\author{
Zhiqin Liu ${ }^{1,2,+}$, Lanping Shi ${ }^{1,2,+}$, Sheng Yang ${ }^{1,2,+}$, Youquan Lin ${ }^{1,2}$, Yahong Weng ${ }^{1,2}$, Xia Li ${ }^{1,2}$, \\ Ansar Hussain 1,2, Ali Noman ${ }^{1,2}$ and Shuilin $\mathrm{He}^{1,2, *}$ \\ 1 Ministry of Education Key Laboratory of Plant Genetic Improvement and Comprehensive Utilization, \\ Fujian Agriculture and Forestry University, Fuzhou 350002, China; lzqfujian@126.com (Z.L.); \\ slpfujian@126.com (L.S.); yangsheng2061@163.com (S.Y.); 18105907468@163.com (Y.L.); \\ wwwyh0915@163.com (Y.W.); lixiatrista@163.com (X.L.); ahtraggar@yahoo.com (A.H.); \\ alinoman@gcuf.edu.pk (A.N.); shlhe201304@aliyun.com (S.H.) \\ 2 College of Crop Science, Fujian Agriculture and Forestry University, Fuzhou 350002, China \\ * Correspondence: shlhe201304@aliyun.com; Tel.: +86-591-8379-3159; Fax: +86-591-8370-4487 \\ + These authors contributed equally to this work.
}

Received: 19 June 2017; Accepted: 30 July 2017; Published: 1 August 2017

\begin{abstract}
Despite the involvement of many members of the chitinase family in plant immunity, the precise functions of the majority of the members remain poorly understood. Herein, the gene ChiIV3 in Capsicum annuum encoding a chitinase protein containing a chitin binding domain and targeting to the plasma membrane was found to be induced by Phytophthora capsici inoculation (PCI) and applied chitin treatment. Besides its direct inhibitory effect on growth of Phytophthora capsici (P. capsici), ChiIV3 was also found by virus-induced gene silencing (VIGS) and transient overexpression (TOE) in pepper plants to act as a positive regulator of plant cell death and in triggering defense signaling and upregulation of $P R$ (pathogenesis related) genes against PCI. A $5^{\prime}$ deletion assay revealed that $p$ ChiIV3 ${ }^{-712}$ to $-459 \mathrm{bp}$ was found to be sufficient for ChiIV3' response to PCI. Furthermore, a mutation assay indicated that W-box ${ }^{-466}$ to -461 bp in $p$ ChiIV3 $3^{-712}$ to $-459 \mathrm{bp}$ was noted to be the PCI-responsible element. These results collectively suggest that ChiIV3 acts as a likely antifungal protein and as a receptor for unidentified chitin in planta to trigger cell death and defense signaling against PCI.
\end{abstract}

Keywords: Capsicum annuum; chitinase; Phytophthora capsici; cell death; promoter

\section{Introduction}

During their life cycle, plants are frequently encountered by multiple types of pathogens and have been armed during their evolution with comprehensive defense mechanisms including two typical interconnecting layers of immunity, termed as pattern triggered immunity (PTI) and effector-triggered immunity (ETI), respectively [1,2]. Common signaling components and sets of pathogenesis related (PR) genes are shared by PTI and ETI, and differ from each other only quantitatively but not qualitatively depending merely on magnitude and duration of the interactions among the signaling components [3,4]. However, the underlying mechanism of PTI and ETI has not yet been fully investigated.

Other than their role as chief constituents in fungi, chitins are also defined as a typical pathogen-associated molecular pattern (PAMP) that is highly conserved among different fungal pathogens. Chitins have been found to be recognized and bound by chitin elicitor receptor kinase 1 (CERK1) or chitin elicitor-binding protein (CeBIP) leading to PAMP-triggered immunity (PTI) [5]. 
In addition, chitins have been found to be targeted and degraded by chitinases resulting in repression of pathogens. Chitinases, encoded by a gene family [6-8], play pivotal roles in plant immunity; for example, the overexpression of a specific chitinase in plants have been frequently found to augment resistance against fungal attacks $[9,10]$. On the other hand, their overexpression often causes obstacles in plant growth and development, possibly due to the competition for available resources for plant growth and development [11]. So the expressions of chitinases are tightly regulated, and plants generally exhibit an inducible chitinase expression at transcriptional level against challenges of pathogens [12-14]. A vast array of cis-elements involved in the response to stress or pathogens have been found in the promoters of chitinase genes [15]. The roles of some of these cis-elements have been investigated in the inducible expression of these genes $[13,14]$. However, the mechanisms underlying the inducible expression of chitinase genes have not been fully understood.

As a Solanaceae, pepper (Capsicum annuum) is a vegetable of great economic importance worldwide. Its growth and yield are curtailed by various soil-borne diseases including Phytophthora blight and bacterial wilt. A better understanding of the immune mechanism will benefit the genetic improvement of disease resistance of pepper. Fifteen putative chitinase genes have been identified in the recently published genomes of pepper. Among these chitinases, class I chitinase [16], CAChi2, a basic class II chitinase [14,17,18], and ChitIV [19] were previously found to be induced by pathogens $[17,18,20,21]$. The -378 CAChi2 promoter was sufficient for CAChi2 gene induction by salicylic acid treatment [14]. In the present study, another member of pepper chitinase genes, designated as ChiIV3, was functionally characterized. We found that ChiIV3 transcripts accumulated in the tissues of pepper leaves upon P. capsici inoculation and exogenously applied chitin treatment. ChilV3 transiently overexpressed in pepper plants triggered intensive hypersensitive response (HR)-like cell death, which accompanied the upregulation of defense-related genes. Conversely, the silencing of ChiIV3 suppressed P. capsici-induced cell death, and enhanced pathogen susceptibility while suppressing the expression of defense-associated genes. $p C_{\text {ChiV }} 3^{-712}$ to $-459 \mathrm{bp}$ was found to be sufficient for ChiIV3's response to PCI, in which the W-box ${ }^{-466}$ to $-461 \mathrm{bp}$ was noted to be the PCI-responsible element. Our data indicate that ChiIV3 is involved in pepper immunity against $P$. capsici inoculation both as a likely antifungal protein and a likely a receptor of chitins.

\section{Results}

\subsection{The Sequence Analysis of ChiIV3 and Its Promoter pChiIV3}

By cDNA-amplified fragment length polymorphism (cDNA-AFLP) analysis, we previously found a TDF (transcript-derived fragment) upregulated due to PCI (Phytophthora capsici inoculation) in pepper. In order to identify its function and underlying mechanism, the corresponding full-length cDNA of ChiIV3 was isolated by the screening of a cDNA library of pepper leaves inoculated with P. capsici. The positive clone was acquired after three rounds of library screening. DNA sequencing showed that the positive clone is a $611 \mathrm{bp}$ cDNA encoding a chitinase of 85 amino acids. A BLAST search found that the putative chitinase gene exhibiting a low degree sequence identities with that of chitinase genes from other plant species. Among the chitinase genes in Arabidopsis, AtChiIV3 (NCBI ID: AT3G54420) showed the highest sequence identity to the positive clone, so it was designated as ChiIV3 (Figure S1). SMART (http://smart.embl-heidelberg.de/) analysis online showed that ChiIV3 contains a CBD (chitin binding domain, amino acids 22-59), indicating that ChiIV3 encodes an extracellular chitinase (Figure S1).

For isolating the corresponding promoter of ChiIV3 ( $p$ ChiIV3), genome DNA walking was performed by using the genome walking kit (Bio S\&T, Saint-Laurent, QC, Canada) with the primers designed according to the ChiIV3 cDNA sequences. A DNA fragment of $1017 \mathrm{bp}$ in length, upstream of the translation start codon, was acquired and the putative cis-element of this promoter was analyzed using PLACE program (http://bioinformatics.psb.ugent.be/webtools/plantcare/html/ and http:/ / www.dna.affrc.go.jp/PLACE/signalscan.html) (Figure S2). Potential regulatory elements 
associated with hormone- and stress-related response were located within the ChiIV3 promoter, including six W-boxes, two heat shock elements (HSEs), two ERELEEs, four CGTCA-motifs, three MYB-related elements, one CCAATBOX1, one TGA-element, and four NODCON1s. The presence of these elements demonstrates that ChilV3 may be involved in pepper's response to biotic and abiotic stresses.

\subsection{ChiIV3 Was Transcriptionally Induced in Pepper Plants against Phytophthora capsici Inoculation (PCI) and Applied Chitin Treatment}

The presence of putative pathogen responsive cis-elements including W-box and CGTCA-motifs in $p$ ChilV3 advocate its probable response to pathogen attack. To test this possibility, qRT-PCR was performed to investigate the transcriptional expression of ChiIV3 detected in pepper leaves inoculated with $P$. capsici spores. The result clearly demonstrated that the transcript level of ChiIV3 can be significantly induced in pepper leaves inoculated with $P$. capsici spores, compared to the mock pepper leaves (Figure 1A). Consistent with this was the promoter activity assay of $p$ ChiIV3 in $p$ ChiIV3:GUS-transiently expressed pepper leaves against the inoculation of $P$. capsici spores. At $24 \mathrm{~h}$ after infiltration of GV3101 cells possessing $p$ ChiIV3:GUS, the leaves were further inoculated with spores of $P$. capsici and harvested at 24 and $48 \mathrm{~h}$ for GUS activity measurement (Figure S3). The results exhibited augmented expression of GUS driven by $p C h i I V 3$ at both $24 \mathrm{hpi}$ (hours post inoculation) and 48 hpi. We also treated the 4-5-week-old pepper plants with applied chitin (typical microbe-associated molecular pattern (MAMP)) and investigated the transcript level of ChiIV3, and the result revealed that transcript level of ChiIV3 can also be significantly enhanced in response to applied chitin treatment (Figure 1B).
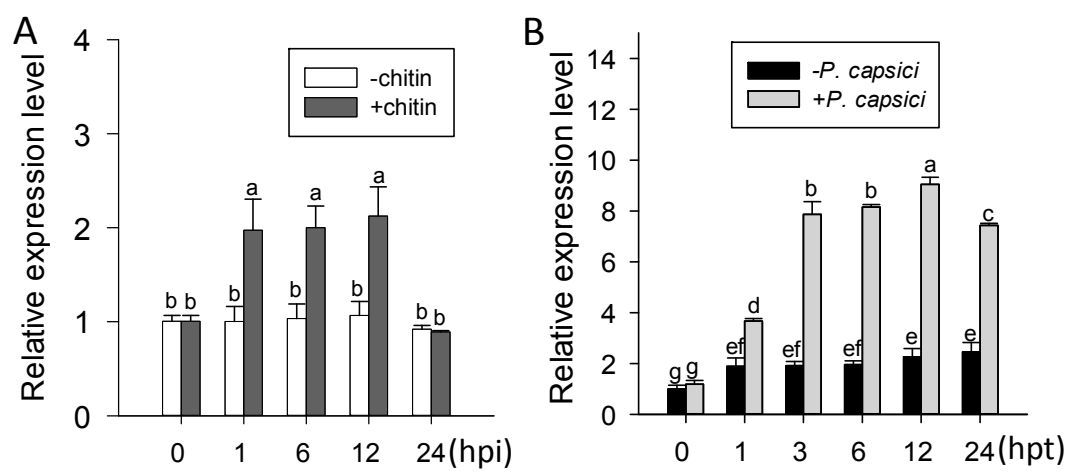

Figure 1. Transcript levels of ChiIV3 in pepper plant in response to exogenously applied chitin treatment and Phytophthora capsici inoculation. (A) The relative transcript levels of ChiIV3 detected in the pepper leaves inoculated with $10 \mu \mathrm{L}$ P. capsici zoospores $\left(\mathrm{OD}_{595}=0.6\right)$ at different time-points. Hpi, hours post inoculation; (B) The transcript level of ChiIV3 can be significantly induced in pepper leaves treated with applied chitin. Hpt, hours post treatment. (A,B) The expression level of untreated plants was set to " 1 ". Values are means \pm SD $(n=3)$. Different letters indicate significant differences determined by Fisher's protected least significant difference (LSD) test $(p<0.05)$.

\subsection{ChiIV3 Protein Is Localized to the Plasma Membrane and Can Be Activated by Applied Chitin Treatment in Nicotiana benthamiana Leaves}

The function of a given protein is closely related to its subcellular targeting. Therefore, unveiling the subcellular localization of ChiIV3 can help us to have a comprehensive description of its function. Keeping this point in view, we generated fused gene vectors of ChiIV3-GFP and SRC2-1-RFP (as a control targeting to the plasma membrane) both driven by the constitutive $35 S$ promoter ( $p 35 S:$ ChilV3-GFP). GV3101 cells containing p35S:ChilV3-GFP and GV3101 cells containing $p 35 S: S R C 2-1-R F P$ were co-infiltrated into the leaves of 7-week-old Nicotiana benthamiana (N. benthamiana) plants [22]. The co-infiltrated leaves were collected for fluorescence detection at 
2 dpi (days post infiltration) with a confocal microscope (Figure 2A). We found that green fluorescent protein) (GFP) signal of ChiIV3-GFP was only observed in the plasma membrane, in accordance with our observation with SRC2-1-RFP. Contrary to the given situation, the signal of GFP in control N. benthamiana leaves was observed in multiple subcellular compartments including the cytoplasm and nuclei. To further elucidate the spatio-temporal regulation of ChiIV3 activation triggered by applied chitin treatment, we constructed a fused gene vector of ChiIV3-GFP driven by its native promoter ( $p C h i I V 3)$ and designated as $p$ ChiIV3:ChiIV3-GFP. We treated the $p C h i I V 3: C h i I V 3-G F P$-transiently expressed $N$. benthamiana leaves with applied chitin. In time-lapse analysis, the fluorescent signals were detected at $12 \mathrm{~h}$ intervals after applied chitin treatment by confocal microscope. The result showed that fluorescent signals of N. benthamiana leaves transiently expressed ChiIV3-GFP driven by its native promoter were observed at the plasma membrane (PM) and the signals can be induced by applied chitin treatment (Figure 2B). The ChiIV3-GFP fluorescent signals in the N. benthamiana leaves were lower in the absence of chitin treatment. Together, these results indicated that ChiIV3 is activated at the PM of $N$. benthamiana cells after applied chitin treatment.
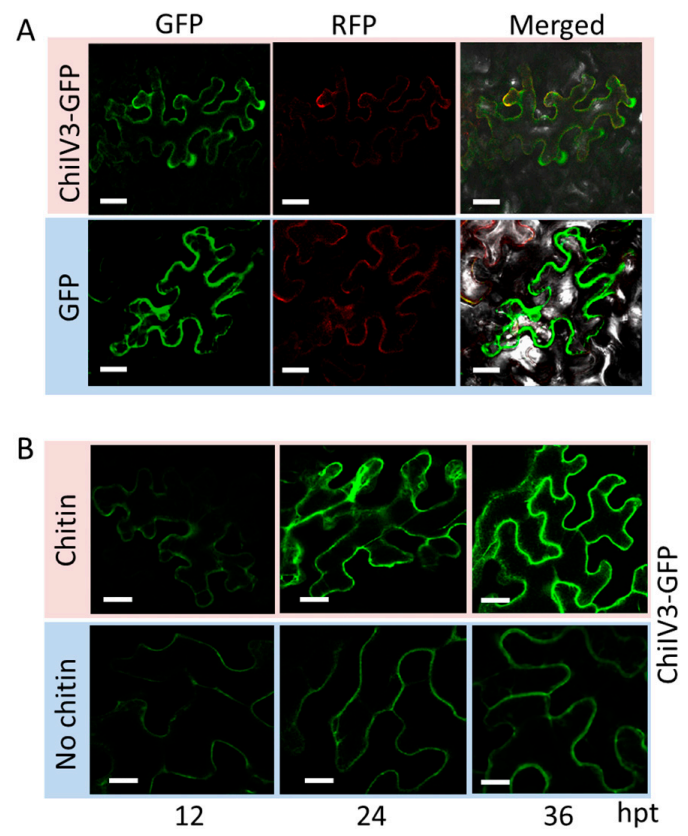

Figure 2. ChiIV3 was located to the plasma membrane and was activated by exogenously applied chitin. (A) Confocal images showed representative N. benthamiana leaf epidermal cells transiently expressing ChiIV3-GFP and SRC2-1-RFP both driven by $35 S$ promoter. The fluorescent signals were detected with the confocal microscope DM6000 CS (Leica, Solms, Germany) at $48 \mathrm{~h}$ after Agro-infiltration. Bars $=50 \mu \mathrm{m}$; (B) ChiIV3 activation during MAMP (chitin) triggered immunity. Time-lapse imaging of pChiIV3:ChiIV3-GFP expressed in N. benthamiana leaves. After chitin treatment, the fluorescent signals were detected at $12 \mathrm{~h}$ intervals. Hpt, hours post treatment. Bars $=50 \mu \mathrm{m}$.

\subsection{Prokaryotic Expressed ChiIV3 Inhibited the Growth of P. capsici Mycelia}

To investigate the possible inhibitory effect of ChiIV3 protein on the growth of P. capsici mycelia, prokaryotic expression of ChiIV3 was performed in Escherichia coli (E. coli) cells. First, ChiIV3-trxA-6HIS fusion protein was expressed in E. coli $4 \mathrm{~h}$ after induction of protein synthesis with isopropyl- $\beta$-D-thiogalactopyranoside (IPTG), and SDS-polyacrylamide gel electrophoresis (SDS-PAGE) assay clearly showed that ChiIV3-trxA-6HIS was successfully expressed in E. coli (Figure 3A). The protein product was purified with HisPur Ni-NTA Superflow Agarose and detected by SDS-PAGE (Figure 3A). Five hundred (500) ng purified ChiIV3 protein was infiltrated into the pepper leaves and obvious cell death was detected, as confirmed by trypan blue staining (Figure 3B). Additionally, $500 \mathrm{ng}$ 
purified ChiIV3 protein was added in the V8 medium cultured mycelia of P. capsici. The $\mathrm{OD}_{595}$ of the V8-cultured P. capsici mycelia was measured at different times after the addition of purified ChiIV3 protein. The result showed, at 6 to $12 \mathrm{~h}$ after the addition of ChiIV3, the increment of $\mathrm{OD}_{595}$ of P. capsici mycelia was significantly suppressed (Figure 3C), compared to that without ChiIV3 protein addition, suggesting a likely antifungal activity of ChiIV3.

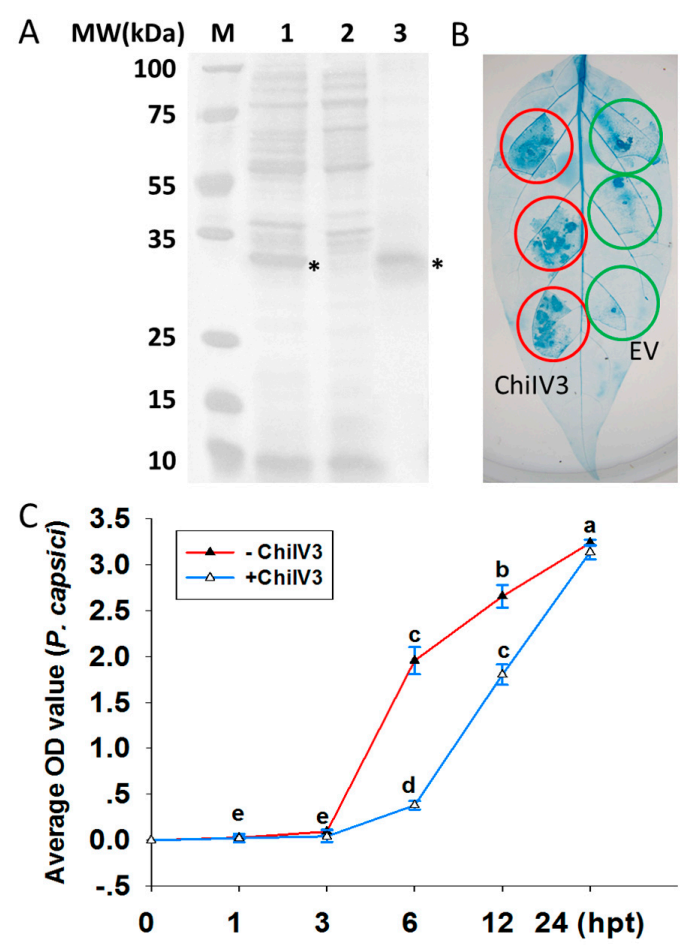

Figure 3. Prokaryotically expressed ChiIV3 inhibited the growth of P. capsici culturing in V8 medium. (A) SDS-PAGE assay was performed to confirm the expression level of ChiIV3 in Escherichia coli cells (BL21). The SDS-PAGE gel was stained with Coomassie Brilliant Blue (CBB) and scanned by a scanner. M, protein ladder marker; 1, pET32a-ChiIV3; 2, pET32a; 3, purified ChiIV3 protein. Asterisks represent the prokaryotically expressed ChiIV3 protein; (B) Trypan blue staining was performed to evaluate the cell death in pepper leaves triggered by purified prokaryotically expressed ChIV3 protein. Red and green circles indicate full and no cell death, respectively; (C) The purified prokaryotically expressed ChiIV3 suppressed the growth of $P$. capsici culturing in V8 medium. The P. capsici was first cultured in V8 medium, followed by supplemented with $500 \mathrm{ng}$ prokaryotically expressed ChiIV3, and the $\mathrm{OD}_{595}$ of $P$. capsici mycelia was measured at different time-points after treatment. Values are means $\pm \mathrm{SD}$ $(n=6)$. Hpt, hours post treatment. Different letters indicate significant differences determined by Fisher's protected LSD test $(p<0.05)$.

\subsection{The Silencing of ChiIV3 Enhanced the Susceptivity of Pepper Plant to P. capsici Inoculation}

To analyze the role of ChilV3 in pepper immunity against P. capsici inoculation (PCI), loss-of-function of ChiIV3 in pepper immunity was assayed by virus-induced gene silencing (VIGS) in pepper plants. The VIGS was performed using the recombinant tobacco rattle virus (TRV) silencing system [23]. A total of 48 ChiIV3-VIGS pepper plants were acquired. We investigated the transcript level of ChiIV3 in ChiIV3-silenced pepper plants challenged with P. capsici to determine the silencing efficiency of ChiIV3. The result was that the transcript level of ChiIV3 in ChilV3-silenced pepper leaves was approximately one-third of that in the unsilenced plants, indicating the success of the silencing (Figure 4A). When inoculated with P. capsici spores, the ChiIV3-silenced plants appeared more prone to disease. In comparison with controlled plants, such susceptibility of silenced plants was manifested by more serious disease symptoms (Figure 4B), faint trypan blue staining (indicator for cell 
death) (Figure 4C) decreased ion leakage (Figure 4D) and lower levels of transcriptional expression of defense-associated marker genes, including DEF1, HIR1, PR1, and BPR1, as well (Figure 4E). Eventually we detected the chitinase activity of ChiIV3-silenced pepper plant in response to PCI; the result indicates that the chitinase of unsilenced pepper leaves was significantly enhanced at $24 \mathrm{~h}$ post-PCI, while that in ChiIV3-silenced plants was partially restrained (Figure 4F). Chitins are known to act as a kind of typical PAMP and can be in planta recognized by pattern recognition receptor (PRR) in resulting plant immunity. To determine whether applied chitin was able to trigger HR-like cell death in pepper leaves and whether the chitin-triggered cell death can be suppressed by ChiIV3-silencing, the ChiIV3-silenced and unsilenced pepper plants were pretreated with applied chitin, and then Fv/Fm and conductivity, respectively, were determined at $24 \mathrm{~h}$ after the chitin treatment to evaluate the HR-like cell death. The result showed that treatment with applied chitin led to a sharp decrease in Fv/Fm and a significant increase in conductivity in the unsilenced pepper plants, while the Fv / Fm decrement and conductivity increment were effectively suppressed in ChiIV3-silenced pepper plants (Figure S4). Taken together, the above results imply that the silencing of ChiIV3 significantly weakened the chitin triggered plant cell death and enhanced the susceptibility of pepper plants to P. capsici inoculation.
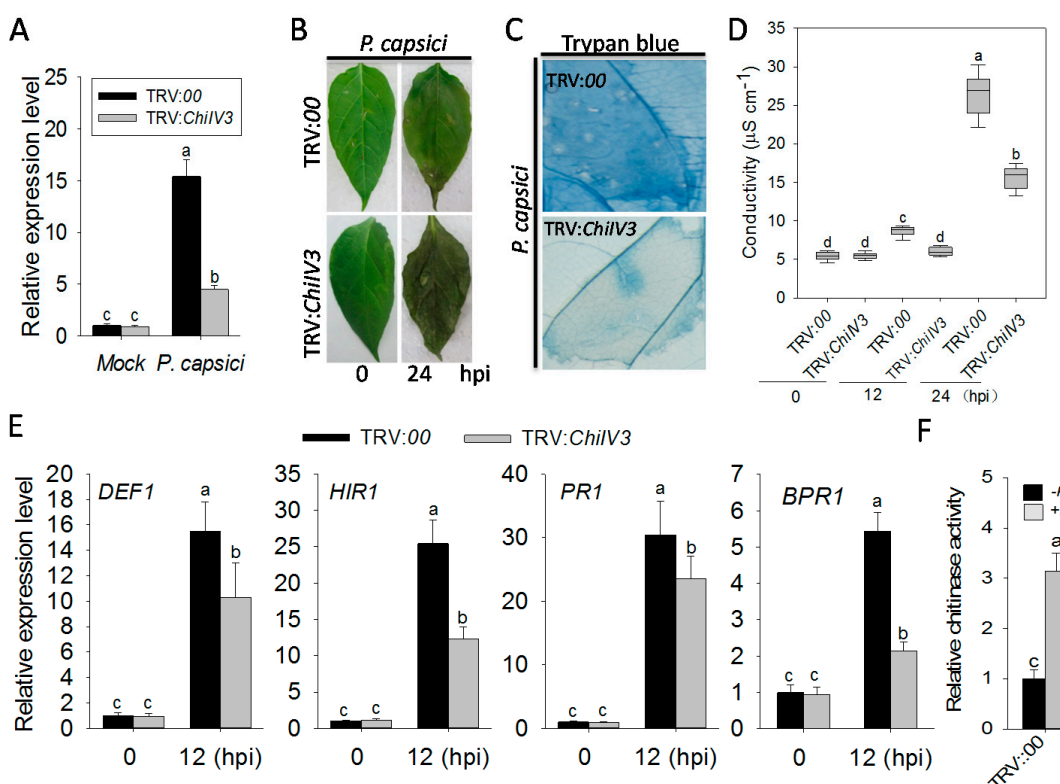

F

Figure 4. The silencing of ChiIV3 by virus-induced gene silencing (VIGS) enhanced the susceptibility of pepper to P. capsici inoculation. (A) Quantitative reverse transcription-PCR (qRT-PCR) analysis of the transcript levels of ChiIV3 in ChiIV3-silenced pepper plants inoculated with P. capsici. The transcript level of ChiIV3 in the mock-treated pepper $\left(\mathrm{ddH}_{2} \mathrm{O}\right)$ leaves was set to " 1 "; $(\mathbf{B})$ The disease symptoms of ChilV3-silenced pepper plants at $24 \mathrm{~h}$ after inoculated with P. capsici spores. Hpt, hours post inoculation; (C) Trypan blue staining and (D) conductivity measurement of ChiIV3-silenced or unsilenced pepper leaves at $24 \mathrm{~h}$ post inoculation of $10 \mu \mathrm{L}$ P. capsici zoospores $\left(\mathrm{OD}_{59}=0.6\right)$. Photos were taken by a camera (Canon) at $24 \mathrm{hpi}$; (E) Determination of the transcript levels of defense-related marker genes in ChilV3-silenced or unsilenced pepper leaves at $12 \mathrm{hpi}$ of $P$. capsici spores, compared to the untreated pepper plants. DEF1, a pepper defensing gene in response to pathogen infection; HIR1, a pepper gene encodes a hypersensitive induced reaction (HIR) protein; PR1, pathogenesis-related protein 1; BPR1, the defense-related genes BPR1 (basic PR-1). The transcript level of all marker genes in unsilenced pepper plants without treatment was set to an expression value of " 1 ". Hpi, hours post inoculation; (F) The relative chitinase activity of ChiIV3-silenced or unsilenced pepper plants at $24 \mathrm{hpi}$ of P. capsici spores. The chitinase activity of unsilenced pepper plants treated with buffer (mock) was set to 1 . $(\mathbf{A}, \mathbf{D}, \mathbf{E})$ Values are means $\pm \mathrm{SD}(n=6)$. Different letters indicate significant differences determined by Fisher's protected LSD test $(p<0.05)$. 


\subsection{Transient Overexpression of ChiIV3 Triggered HR-Like Cell Death and Enhanced the Expression of Immunity Associated Marker Genes}

To confirm the results from ChilV3-silencing by VIGS, we performed a gain of function experiment employing Agrobacterium-mediated transient overexpression in pepper plants, which have been frequently used in the functional genomic studies of pepper [24,25]. GV3101 cells containing p35S:ChiIV3 (p35S:00 was used as negative control) were infiltrated into leaves of pepper (Capsicum annuum cv Yanshan01, with moderate Phytophthora blight resistance). Immunoblot assay showed that ChiIV3 protein was successfully expressed in pepper leaves at 48 hpi (Figure 5A). An intensive cell death phenotype was observed in the ChiIV3 transient overexpressing pepper leaves coupled with brownish color in 3,3'-Di-aminobenzidine (DAB) staining (indicator for $\mathrm{H}_{2} \mathrm{O}_{2}$ accumulation) and darker trypan blue (Figure 5B), higher level of ion leakage (Figure 5C), higher chitinase content in pepper leaves (Figure 5D), as well as elevated levels of transcriptional expression of immunity associated marker genes, including DEF1, HIR1, PR1, and BPR1 measured by qRT-PCR (Figure 5E). On the basis of the close consistency between loss of function and gain of function, it is strongly proposed that ChiIV3 plays a vital role in the induction of plant cell death and pepper immunity in response to $P$. capsici.

\subsection{A Region from $-459 \mathrm{bp}$ to $-712 \mathrm{bp}$ in the Promoter of ChiIV3 Is Adequate for the PCI-Response of ChilV3}

The inducible expression of ChilV3 against PCI is supposed to be conferred by its promoter through binding by specific transcription factors (TFs). To determine the crucial promoter region and the possible cis-elements responsible for the response of $p C h i l V 3$ to $P$. capsici inoculation, $5^{\prime}$ deletion assay was conducted. Based on the distribution of cis-elements possibly responsible for biotic and abiotic responses, five $5^{\prime}$ deletions of $p$ ChiIV3 beginning from $-1017,-891,-712,-459$, and -276 $\mathrm{bp}$ to the initiation codon were amplified and cloned into upstream of GUS reporter gene in the destination vector pDMC163 (Figure 6A). EHA105 cells containing the generated constructs above were transformed into tobacco plants to get the $T_{0}$ plants and their corresponding $T_{1}, T_{2}$, and $T_{3}$ lines. With the plants of homozygous $\mathrm{T}_{3}$ tobacco lines, the promoter region responsible for $P$. capsici response was assayed with GUS activity quantification against $P C$ inoculation (Figure 6B). We observed that $-1017,-892$, and -712 bp deletions were sufficient to trigger the GUS expression under P. capsici challenge, while GUS expression driven by the -459 and -276 bp deletions failed to be induced by $P C$ inoculation. The result demonstrated that the promoter region located between -712 and $-459 \mathrm{bp}$ is essential for the induction of ChilV3 in response to P. capsici inoculation.

\subsection{W5 Was Found to Be the Only W-Box Conferring the Response of pChiIV3 to PCI}

The PCI-responsive promoter region noticeably contains three $\mathrm{W}$-boxes, including W3 $\left(\mathrm{W}^{-672 \text { to }-667}\right), \mathrm{W} 4\left(\mathrm{~W}^{-640}\right.$ to -635$)$, and $\mathrm{W} 5\left(\mathrm{~W}^{-466}\right.$ to -461$)$, while six $\mathrm{W}$-boxes were included in the full-length promoter (W1-W6) (Figure 7A). To determine the exact W-box associated with PCI, the $p$ ChiIV3 with mutated W3, W4, or W5 was constructed by site-directed mutagenesis, according to the previous study described by Colleen Knoth and Thomas Eulgem [26]. Mutated constructs were fused to GUS in a reporter vector to generate $p$ ChiIV3-W3m:GUS, $p$ ChiIV3-W4m:GUS, and $p$ ChiIV3-W5m:GUS and transformed into Agrobacterium GV3101 cells individually (Figure 7B-D). These cells containing each reporter vector were infiltrated into leaves of 8-week-old pepper plants. At $24 \mathrm{~h}$ after infiltration, the leaves were inoculated with spores of $P$. capsici. The pathogen inoculated leaves were harvested for GUS activities quantification assay at $48 \mathrm{hpi}$. The result depicted no effect of the mutation of W3 and W4 upon the expression of GUS in response to PCI (Figure 7B,C) while that of the mutation of W5 considerably downregulated the expression of GUS (Figure 7D). From the observations, we concluded that W5 has a vital role in $p$ ChiIV3 against $P$. capsici inoculation. 

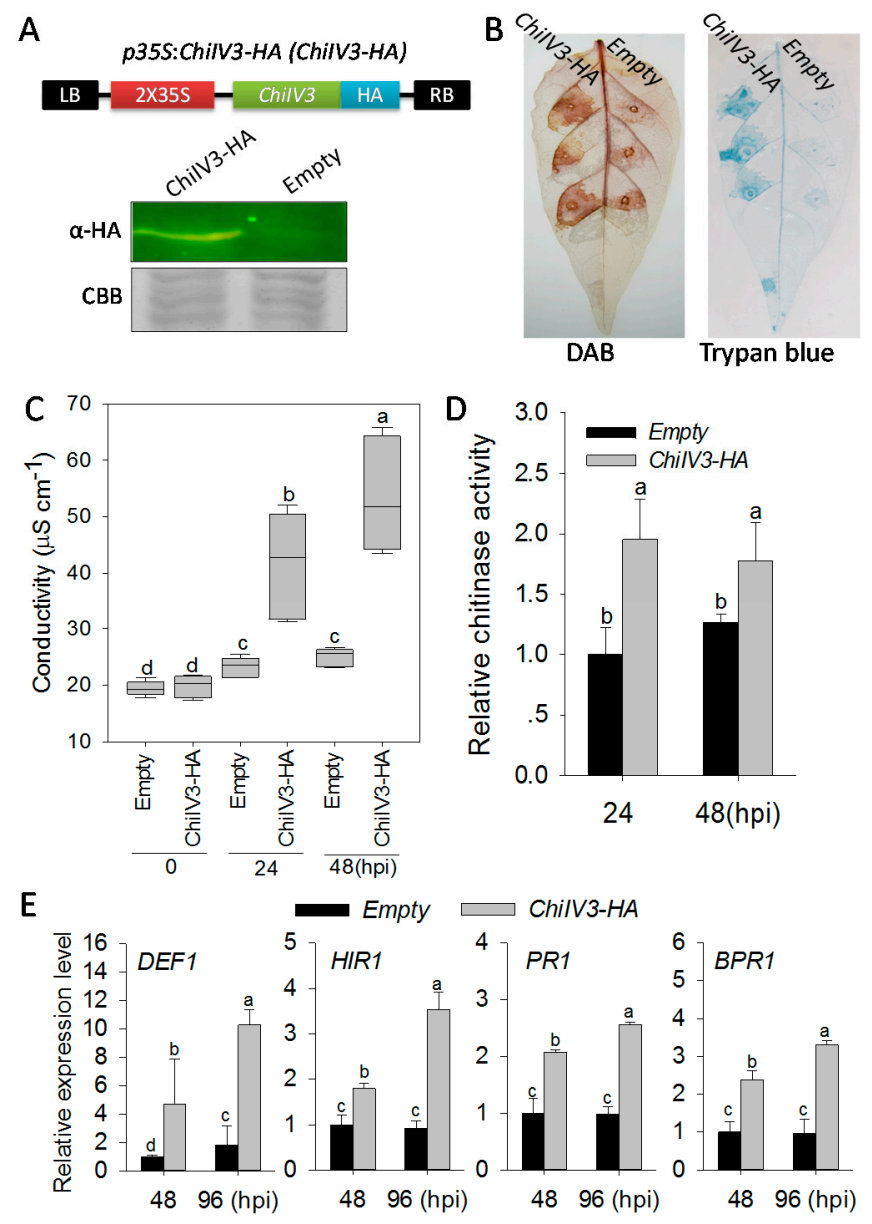

Figure 5. Transient overexpression of ChiIV3 triggered intensive cell death in pepper leaves. (A) The schematic diagram of the p35S:ChiIV3-HA vector and the immunoblotting analysis of ChiIV3-HA transiently expressed in pepper leaves. An anti-HA antibody was used to detect ChiIV3-HA fused protein in the immunoblotting analysis. CBB was used to normalize the total protein levels. $2 \times 35 \mathrm{~S}$, two copies of the cauliflower mosaic virus $35 \mathrm{~S}$ promoter; LB, left border; RB, right border; CBB, Coomassie Brilliant Blue; $\alpha$-HA, $\alpha$-hemagglutinin (HA) antibody; (B) The DAB and trypan blue staining of pepper leaves transiently overexpressed ChiIV3-HA (p35S:ChiIV3-HA) at $48 \mathrm{hpi}\left(\mathrm{OD}_{595}=0.6\right)$. The experiment was performed three times independently with similar results obtained each time. DAB, DAB staining; Trypan blue, trypan blue staining; (C) Conductivity measurement of discs of pepper leaves infiltrated with GV3101 cells harboring p35S:ChiIV3-HA or p35S:00 (control). Hpi, hours post infiltration; (D) The relative chitin content of pepper leaves transiently overexpressed ChiIV3-HA. The chitin content of the control pepper leaves (expressed p35S:00) was set to " 1 ". Hpi, hours post infiltration; (E) Quantification of transcript levels of defense-related marker genes in ChiIV3-HA transiently overexpressed pepper leaves at different time-points. DEF1, a pepper defensing gene in response to pathogen infection; HIR1, a pepper gene encodes a hypersensitive induced reaction (HIR) protein; PR1, PATHOGENESISRELATED PROTEIN1; BPR1, the defense-related genes BPR1 (basic PR-1). The transcript levels of the marker genes in the control pepper leaves (transiently expressing p35S:00 at 48 hpi was set to "1". Hpi, hours post infiltration; (C-E) Values are means \pm SD $(n=6)$. Error bars indicate SD. Different letters indicate significant differences determined by Fisher's protected LSD test $(p<0.05)$. 


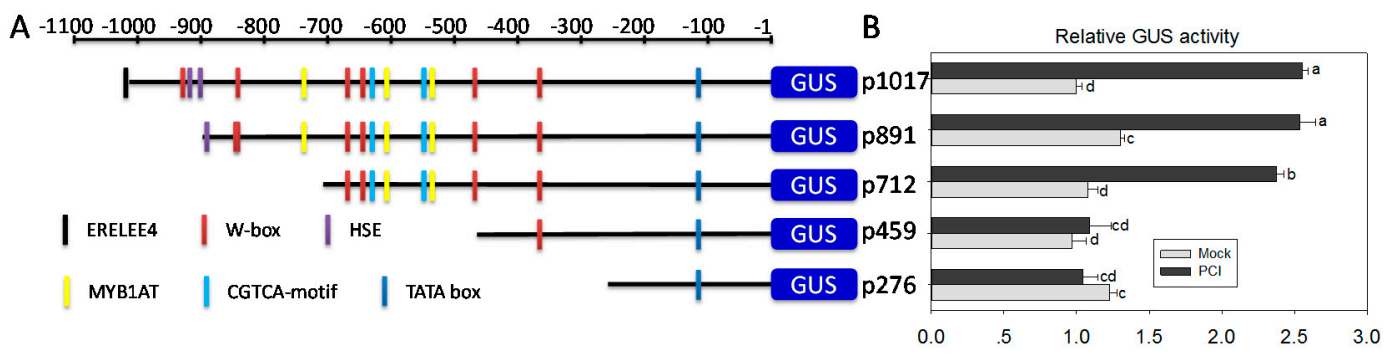

Figure 6. Deletions analysis of ChiIV3 promoter in response to P. capsici inoculation. (A) Schematic diagram of the reporter vector constructs with serial 5'-deletions of ChiIV3 promoter fused to the GUS reporter gene. The serial $5^{\prime}$ deletions of ChiIV3 promoter were fused to the GUS reporter gene in the vector pMDC163 and transformed into GV3101 for tobacco transformation. Seedlings of T3 lines were used for GUS activity assay. Putative cis-elements in ChiIV3 promoter were represented by boxes with different colors; (B) The expression of GUS driven by the five deletions in plants of T3 tobacco lines in response to $P$. capsici inoculation. The activity of GUS driven by 1017 with mock treatment was set to " 1 ". Values are means $\pm \mathrm{SD}(n=6)$. Different letters indicate significant differences determined by Fisher's protected LSD test $(p<0.05)$.

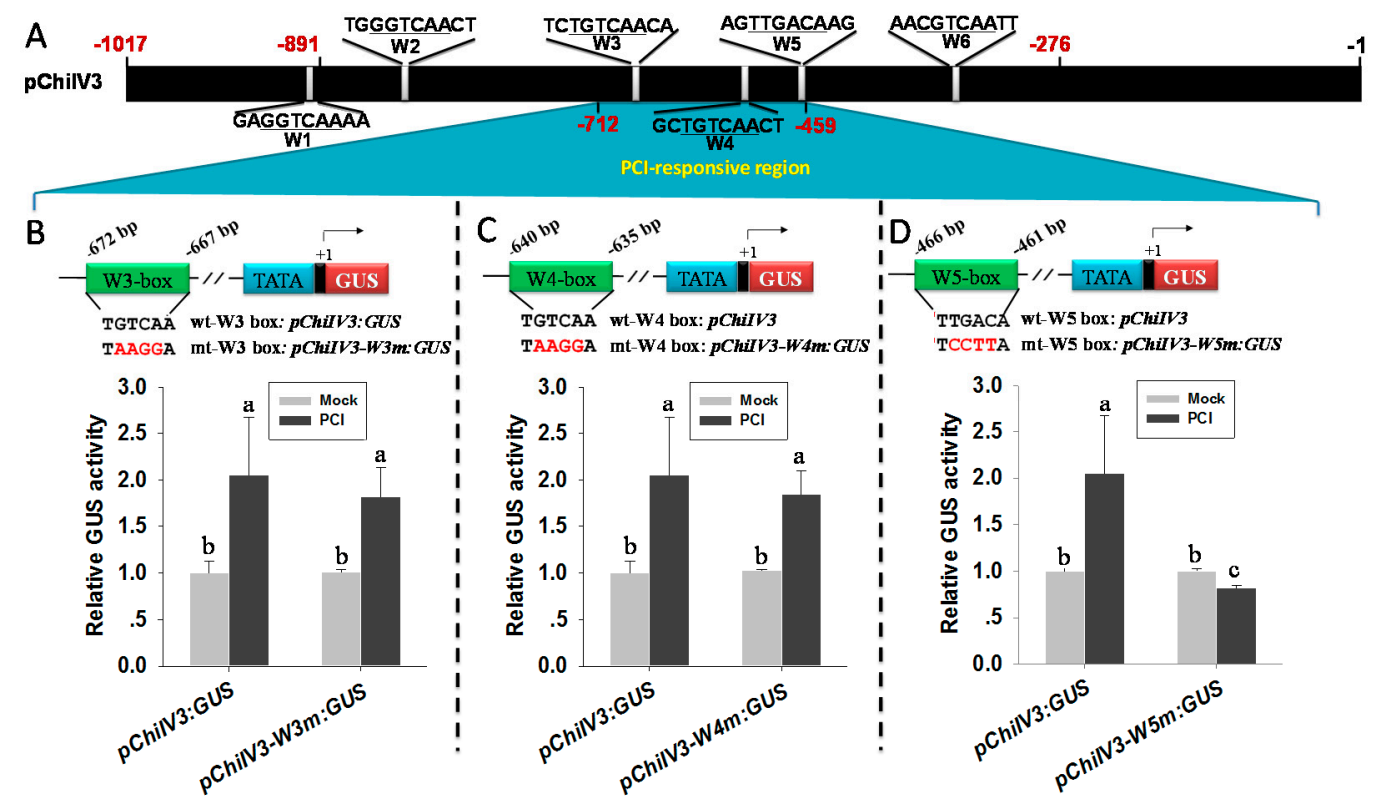

Figure 7. Site-directed mutagenesis analysis of ChilV3 promoter in response to $P$. capsici inoculation. (A) The W-box locations in ChiIV3 promoter. The positions of the deletions are indicated by red numbers, and each W-box is indicated by a white box. The underlined nucleotides represent the core sequence of W-box; (B-D) The schematic diagram of $p$ ChiIV3:GUS and its W-box-mutant constructs ( $p$ ChiIV3-W3m:GUS, pChiIV3-W4m:GUS, or pChiIV3-W5m:GUS, the mutated nucleotides of W-box are in red color) and the activities of GUS driven by $p$ ChilV3:GUS and its three W-box-mutant in response to $P$. capsici inoculation in pepper leaves tissues transiently transformed with $p$ ChiIV3:GUS or its W-box-mutant constructs, respectively. The GV3101 cells containing the pChiIV3:GUS or its W-box-mutant constructs were vacuum-infiltrated into the pepper leaves and maintained in the greenhouse for $24 \mathrm{~h}$. The infiltrated plants were then treated with P. capsici spores. The $P$. capsici-inoculated leaf tissues were harvested for GUS activity measurements at 2 dpi. Values are means $\pm S D(n=6)$. Error bars indicate SD. Different letters indicate significant differences determined by Fisher's protected LSD test $(p<0.05)$. 


\section{Discussion}

Chitinases constitute a family of common antifungal proteins, which function against fungal pathogens through degrading chitin in both PTI and ETI $[27,28]$. To function properly, the expression of chitinase genes must be securely regulated. However, the precise functions of individual members in this family and their expressional mechanism remain largely unknown. Our data in the present study provide strong evidence that pepper ChiIV3 not only acts as a likely antifungal protein but also as a likely receptor of an unidentified chitin in triggering defense signaling and pepper immunity.

A key step in both PTI and ETI is the transcriptional reprogramming of a multitude of defense-associated genes through specific binding of their cis-elements in promoters by corresponding TFs [29-33]. So, the presence of cis-elements in the promoter of a given immunity associated gene might be closely related to their expression and therefore functions in plant immunity. In $p$ ChiIV3, putative pathogen responsive elements including W-box [34-36], TGA-box [37], and CGTCA-box (MeJA responsive element) [38] have been implicated in defense responses. The presence of these cis-elements supports our speculation that ChiIV3 might respond to pathogen attack. Consistently, our data showed that ChiIV3 was transcriptionally regulated by exogenously applied chitin as well as PCI, which was further confirmed by the inducible expression of GUS driven by $p$ ChilV3 by P. capsici inoculation in pepper plants. These findings imply a role of ChiIV3 in pepper immunity against $P$. capsici, since genes upregulated during plant immune responses have been frequently found to play important roles in disease resistance [39-42]. This speculation was further confirmed by the data that the purified prokaryotically expressed ChiIV3 protein in E. coli exhibited a likely antifungal activity to the growth of $P$. capsici mycelia from 3 to $12 \mathrm{~h}$ after its addition. In addition, the silencing of ChiIV3 by VIGS significantly suppressed MAMPs (chitin) triggered plant cell death and enhanced the susceptivity of pepper plants to $P$. capsici inoculation, manifested by severe disease symptom, increased ion leakage, and paler trypan blue staining compared to the mock, accompanied by lower levels of transcriptional expression of immunity-associated marker genes including DEF1, HIRI, PR1, and BPR1. By contrast, in ChiIV3 transiently overexpressing pepper leaves, a darker trypan blue and DAB staining were found coupled with enhanced expression of DEF1, HIRI, PR1, and BPR1, indicating that ChiIV3 is a positive regulator in plant cell death. All these data advocate that besides the direct suppression of P. capsici, ChiIV3 also acts as a likely receptor in the plasma membrane to an unidentified chitin to activate immune signaling components including $\mathrm{H}_{2} \mathrm{O}_{2}$ and expression of $\mathrm{PR}$ genes. The GFP signals of ChiIV3-GFP transiently overexpressed cells of $N$. benthamiana leaves were exclusively observed in plasma membrane, and the ChiIV3 activation can be efficiently induced by applied chitin treatment. Similarly, a class IV chitinase ChitIV was recently found to play a role in defense signaling by interacting with receptor-like cytoplasmic protein kinase PIK1 [19]. Chitins are generally believed to be typical fungi based PAMPs and are directly targeted by chitin elicitor receptor kinase 1 (CERK1) or Chitin elicitor-binding protein (CeBIP) leading to PAMP-triggered immunity (e.g., PTI) [5]. We speculate that some chitinases inclusive of ChiIV3 might have been co-opted for recognition of chitins to trigger downstream defense signaling, but more investigations and further evidence are still required. As $P$. capsici is an oomycete, whose cell wall is believed to be made of cellulose but not chitin, how genes encoding chitinases such as ChiIV3 were induced by P. capsici and how they function in the defense reaction against $P$. capsici remains undetermined at this point in time. Similar observations have also been published previously that CAChi2 was significantly induced and accumulated not only in pepper tissues but also in the cell surface of $P$. capsici $[17,18]$. It was speculated that CAChi2, induced by elicitors of $P$. capsici, might be able to hydrolyze the cell wall of $P$. capsici through a mechanism that remains to be determined $[17,18]$. So, chitinases are generally induced by attack of $P$. capsici in pepper plants and are involved in the immunity against $P$. capsici, although the underlying mechanism remains to be elucidated in the future.

To further elucidate the molecular basis of the inducible expression of ChiIV3 in response to $P$. capsici infection, a $5^{\prime}$ deletions assay of $p C h i I V 3$ in stable transgenic tobacco plants and site-directed mutagenesis was performed to determine the promoter region and the exact cis-element conferring the 
inducible expression of ChiIV3 against $P$. capsici infection. The results indicate that the promoter region

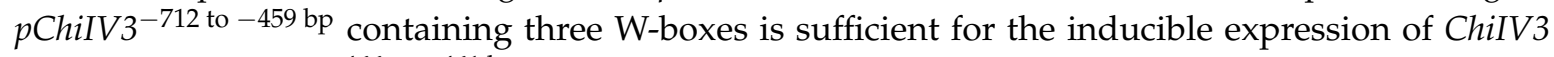
against PCI, and $\mathrm{W}^{-466}$ to $-461 \mathrm{bp}$ is the only W-box responsible for its response to PCI. Given that WRKYs, which bind to W-box and constitute a large TF family in plants [34], play an important roles in the regulation of plant immunity [29,43-45], we speculate that the inducible expression of ChiIV3 to P. capsici inoculation is conferred by $\mathrm{W}^{-466}$ to $-461 \mathrm{bp}$ via its binding to specific unidentified WRKY TF. Further identification and functional characterization of the WRKY TF recognizing and binding to this W-box would provide new insight into immunity mediated by ChiIV3.

Collectively, our data strongly suggest that ChiIV3 not only likely acts as an antifungal protein but also as a receptor of chitins, since both the exogenous application of ChiIV3 and its transient overexpression triggered cell death and expression of PR genes in pepper plants, and the $\mathrm{W}^{-466}$ to $-461 \mathrm{bp}$ is responsible for the inducible expression of ChiIV3 against PCI. The results in the present study will benefit the further dissection of pepper immunity against $P$. capsici as well as its genetic improvement.

\section{Materials and Methods}

\subsection{Plant Material and Plant Cultivation}

Seeds of pepper inbred line yanshan-01 (Capsicum annuum cv Yanshan01), tobacco cultivar K326 (Nicotiana tabacum, used for transformation), and N. benthamiana (used for transient overexpression assay) were collected by the pepper breeding group in Fujian Agriculture and Forestry University (http:/ / www.fafu.edu.cn). Pepper, tobacco, or N. benthamiana seeds were germinated in stream-sterilized soil mix (peat moss: per liter $(2: 1, v / v)$ ) in plastic pots. Two weeks after germination, the plants were transferred to larger pots and maintained in a growth room under a condition of $25^{\circ} \mathrm{C}$, 60-70 mmol photons $\mathrm{m}^{-2} \cdot \mathrm{s}^{-1}$, a relative humidity of $65-70 \%$, and a $16 \mathrm{~h} / 8 \mathrm{~h}$ light/dark photoperiod.

\subsection{Pathogen Inoculation}

The P. capsici strain used in this study was isolated from Phytophthora blight infected pepper plants collected from Huian County, Fujian Province, China. For preparation of zoospores, P. capsici was cultured in V8 medium (200 g. $\mathrm{L}^{-1}$ tomato juice, $\left.3 \mathrm{~g} \cdot \mathrm{L}^{-1} \mathrm{CaCO}_{3}\right)$ overnight at $28^{\circ} \mathrm{C}$ and collected with low speed centrifugation suspended in sterile $\mathrm{ddH}_{2} \mathrm{O}$. Suspension was then adjusted to a density of 100 zoospores $\mathrm{mL}^{-1}$. For inoculation, the tobacco or pepper leaves were inoculated with $10 \mu \mathrm{L}$ of the zoospore suspension by leaflet cutting (perpendicular to the midrib of leaflet, deep cut at two-thirds to the midrib) using a syringe with a needle, and the leaves were harvested at different time points for further assay. Control plants were inoculated with sterile $\mathrm{ddH}_{2} \mathrm{O}$.

\subsection{Isolation of ChiIV3 Promoter}

Genomic DNA used for the isolation of pChiIV3 was extracted from pepper leaves using the hexadecyl trimethyl ammonium bromide (CTAB) method [46]. The promoter sequences of ChiIV3 upstream of the translation start codon was amplified using the APA gene Genome Walking Kit (Bio S\&T, Saint-Laurent, QC, Canada; http:/ / www.biost.com/page/default.aspx) with three nested ChiIV3 antisense gene-specific primers, including ChiIV3-GSPa, ChiIV3-GSPb, and ChiIV3-GSPc (see Supplemental Table S1). Firstly, single-strand DNA (ssDNA) fragments were produced from the pepper genomic DNA by a single primer extension reaction using ChiIV3-GSPa. Then, degenerate random tag (DRT) primer was added to the amplified ssDNA product to generate the double-strand DNA in the second PCR. Later, ChiIV3-GSPb and ChiIV3-GSPc were used in two rounds of nested PCR, respectively. Finally, the amplified product was cloned into the pMD-18T vector (TaKaRa, Tokyo, Japan, http://www.takara.com) and sequenced. The acquired DNA was 1017 bps in length and the cis-acting elements of $p C h i I V 3$ were analyzed online using the softwares PLACE 
(http:/ / www.dna.affrc.go.jp/PLACE/signalscan.html) and PLANTCARE (http:/ / bioinformatics.psb. ugent.be/webtools/plantcare/html) [47,48].

\subsection{Amplification of $5^{\prime}$ Deletions or Site-Directed Mutagenesis of $p$ ChiIV3 by PCR}

$p$ ChiIV3 and its $5^{\prime}$ deletion derivatives $(-1017,-891,-712,-833$, and -447 to +1 ; the first nucleotide A in the initiation codon ATG was set as +1 ) were constructed using high-fidelity PCR (GXL DNA Polymerase; TaKaRa, Tokyo, Japan,) with Gateway-compatible primers flanking the attB sequence (see Supplemental Table S1). Site-directed mutagenesis of the $p$ ChiIV3 was performed using primers $p$ ChiIV3-W3m-F, $p$ ChiIV3-W4m-F, and $p$ ChiIV3-W5m-F (see Supplemental Table S1). The attB-containing PCR product of $p C h i I V 3$ and its $5^{\prime}$ deletion derivatives were introduced into the pDONR207 via BP reaction. After confirmation by sequencing, the BP products were transferred into the destination vector pMDC163 via LR reaction to generate p1017, p891, p712, p458, p276, respectively. All the generated constructs above were introduced in GV3101 for further assay.

\subsection{Vector Construction by Gateway Cloning Technique}

The vector p35s:ChiIV3-GFP and pChiIV3:ChiIV3-GFP for subcellular localization assay, TRV2:ChiIV3 for virus induced gene silencing, p35S:ChiIV3, pChiIV3-serial-deletions:GUS, pChiIV3-W3m:GUS, $p$ ChiIV3-W4m:GUS, $p$ ChiIV3-W5m:GUS for promoter activity assays for transient overexpression in pepper plants were all constructed by Gateway cloning technique (Invitrogen; http:/ / www.invitrogen.com). The full cDNA or promoter regions were amplified using high-fidelity PCR (GXL DNA Polymerase; TaKaRa, Tokyo, Japan) with Gateway-compatible primers flanking the attB sequence (see Supplemental Table S1). To construct vector W5-p35S core:GUS or W5m-p35S core:GUS, the DNA fragment containing W5 box (or W5m) fused to the core CaMV35S promoter ( -46 to +8 ) and flanked by $5^{\prime}$ and $3^{\prime}$ termini with attB were synthesized by Convenience Corporation (Suzhou, China). All of the PCR amplified or synthesized attB containing DNA fragments above were cloned into satellite vector pDONR207 (Invitrogen, Carlsbad, CA, USA) by BP reaction (Invitrogen). Confirmed by sequencing, the BP products above were then transferred to various Gateway compatible destination vectors such as pMDC83, pTRV2, pMDC163, and pEarleyGate201 [49]. To construct pET32a-ChiIV3 for prokaryotic expression, the open reading frame of ChiIV3 was amplified by PCR with primers flanking with appropriate restriction enzyme cutting sites (Bam H I and Xho I) and were further cloned in frame to the expression cassette in pET32a to generate pET32a-ChiIV3. Confirmed by sequencing, the pET32a-ChiIV3 was introduced into the E. coli BL21 for prokaryotic expression.

\subsection{Tobacco Transformation}

The method described by Muller et al. [50] was used to generate the transgenic tobacco plants. To do this, EHA105 agrobacterium cells containing the corresponding serial deletion vectors were used to infect tobacco leaf discs for transformation. The potential tobacco transformants were screened on Murashige \& Skoog (MS) medium supplemented with $500 \mathrm{mg} / \mathrm{L}$ carbenicillin and with $75 \mathrm{mg} / \mathrm{L}$ hygromycin (Roche; http://www.roche.com). Additionally, the regenerated plants were further confirmed by PCR with specific primers. Later these plants were self-pollinated to acquire the seeds of T1 lines screened with $75 \mathrm{mg} / \mathrm{L}$ hygromycin during their germination. The resulting plants were self-pollinated again to acquire the seeds of T2 lines. Plants of T3 lines were selected and used for $p C h i I V 3$ activity assay.

\subsection{Prokaryotic Expression of ChiIV3}

To induce the expression of ChilV3 protein, the construct $p E T 32 a-C h i I V 3$ was introduced into $E$. coli BL21 cultivated in Luria Bertani (LB) media. IPTG $(1 \mathrm{mM})$ was added and cells were harvested $4 \mathrm{~h}$ after the addition of IPTG. Following treating with $1 \mathrm{mg} / \mathrm{mL}$ lysozyme, the suspension ChiIV3 protein was incubated on ice for $1 \mathrm{~h}$ and then sonicated for $1 \mathrm{~h}$ to break the cells. The prokaryotic expressed 
ChiIV3 was purified with HisPur Ni-NTA Superflow Agarose (Thermo; http:/ / www.thermo.com.cn) and detected by SDS-PAGE.

\subsection{Chitinase Activity Measurement}

A chitinase activity measurement was performed as previously described [51], with slight modification. Colloidal chitin was prepared as described by Hsu and Lockwood [52]. Total proteins from ChilV3-transiently expressed or ChilV3-silenced or untreated pepper were extracted using the protein extraction buffer $(10 \%$ glycerol, $25 \mathrm{mM}$ Tris- $\mathrm{HCl}, \mathrm{pH} 7.5,150 \mathrm{mM} \mathrm{NaCl}, 1 \mathrm{mM}$ ethylene diamine tetraacetic acid (EDTA), 2\% Triton X-100, $10 \mathrm{mM}$ DTT, $1 \times$ complete protease inhibitor cocktail (Sigma-Aldrich, Saint Louis, MO, USA, http://www.sigma.com) and 2\% (w/v) polyvinylpolypyrrolidone) [25]. An equal amount of total proteins above were incubated with colloidal chitin at $45^{\circ} \mathrm{C}$ for $1 \mathrm{~h}$. The reaction was terminated by adding $100 \mu \mathrm{L} \mathrm{HCl}(1 \mathrm{~N})$ on ice and incubating for $10 \mathrm{~min}$. The reaction solution was centrifuged for $10 \mathrm{~min}$ at a speed of $13,000 \mathrm{rpm}$ to precipitate the undigested substrate. The generated $\mathrm{N}$-acetyl glucosamine residue was spectrophotometrically measured by the dinitrosalycylic acid (DNSA) method described by Miller [53].

\subsection{The Maximal Photochemical Quantum Efficiency of Photosystem II}

The maximal photochemical quantum efficiency of photosystem II was detected as Li previously described [54] with slight modification. Twenty-four hours after treatment, the stress tolerance of the applied chitin treated ChiIV3-silenced or unsilenced pepper leaves were measured on the basis of changes in the maximal photochemical efficiency of photosystem II. Chlorophyll fluorescence was determined with imaging pulse amplitude modulation fluorometer (IMAG-MAXI; Heinz Walz).

\subsection{Fluorometric Assays for GUS Activity}

For GUS activity quantification, the total proteins of stress-treated leaves in transgenic tobacco or pepper transiently expressed the GUS-tag vectors were extracted according to the method of Choi du et al. [25] with slight modification. The rate of $p$-nitrophenol $(\gamma=415 \mathrm{~nm})$ release was measured by using a microplate reader (Biotek, Winooski, VT, USA, http:/ / www.biotek.com) to measure GUS activity.

\subsection{Agrobacterium-Mediated Transient Expression Assay}

The recombinant vectors were introduced into Agrobacterium strain GV3101 by the freeze/thaw method. Agrobacterium-mediated transient expression assays of different genes or promoters were performed in pepper plants according to the method described by Liu with slight modification [26]. Agrobacterium cells harboring different constructs were grown in YEP medium $(10 \mathrm{~g} / \mathrm{L}$ yeast extract; $10 \mathrm{~g} / \mathrm{L}$ peptone; $5 \mathrm{~g} / \mathrm{L}$ sodium chloride; $\mathrm{pH} 7.0$ ) overnight at $28^{\circ} \mathrm{C}$ with shaking, and agrobacterium cells were collected by centrifugation $(7500 \times g$ at room temperature for $10 \mathrm{~min})$, and suspended in the infiltration medium (10 mM MgCl $2,10 \mathrm{mM} \mathrm{MES,} \mathrm{pH} \mathrm{5.7,} 200 \mu \mathrm{M}$ acetosyringone). The suspension cells were infiltrated into pepper leaves using a needleless syringe. The plants were maintained in a greenhouse at $25^{\circ} \mathrm{C}$.

\subsection{Western Blotting Assay}

Western blotting was performed following the method used in our previous study (Liu et al. 2015). Proteins used in Western blotting were isolated with extraction buffer $(10 \%$ glycerol, $25 \mathrm{mM}$ Tris- $\mathrm{HCl}$, pH 7.5, $150 \mathrm{mM} \mathrm{NaCl}, 1 \mathrm{mM}$ EDTA, $2 \%$ Triton X-100, $10 \mathrm{mM}$ DTT, $1 \times$ complete protease inhibitor cocktail (Sigma-Aldrich; http://www.sigma.com) and 2\% (w/v) polyvinylpolypyrrolidone [25]). SDS-PAGE was performed to separate the proteins with different sizes. The rabbit anti-HA-peroxidase antibody (Abcam, Cambridge, UK, http:/ / www.abcam.com) and goat anti-rabbit IRDye 800CW (Odyssey li-Cor, Cambridge, UK, http:/ /www.licor.com) were used to detect the protein level of HA-fused protein. 


\subsection{Subcellular Localization}

Agrobacterium tumefaciens stains GV3101 harboring p35S:ChilV3-GFP, p35S: GFP and p35S:SRC2-1-RFP (used as a control targeting to the plasma membrane) were recovered via culturing two times. Recovered GV3101 cells containing p35S:ChilV3-GFP and p35S:SRC2-1-RFP were mixed at a ratio of 1:1 and co-infiltrated into $N$. benthamiana leaves using a syringe without a needle. At appropriate timepoints, the fluorenscence of the $N$. benthamiana leaves were detected using a laser scanning confocal microscope (TCS SP8, Leica, Solms, Germany).

\subsection{Total RNA Isolation and Quantitative Real-Time PCR Analysis}

To determine the relative transcriptional levels of targeted genes, real-time PCR was performed with specific primers (see Supplemental Table S2) according to the manufacturer's instructions for the SYBR Premix Ex Taq II system (TaKaRa, Tokyo, Japan) and the Bio-rad Real-time PCR system (Bio-rad, Hercules, CA, USA, http:/ / www.bio-rad.com). Total RNA preparation and qRT-PCR were carried out following procedures adopted in our previous studies [55-57]. Four independent biological replicates of each treatment were performed. Data were analyzed by the Livak method [58] and expressed as a normalized relative expression level $\left(2^{-\Delta \Delta C t}\right)$ of the respective genes. The relative transcript level of each sample was normalized by CaActin and 18S ribosomal RNA (EF564281), respectively.

Supplementary Materials: Supplementary materials can be found at www.mdpi.com/1422-0067/18/8/1661/s1.

Acknowledgments: We would like to thank Zonghua Wang (Key Laboratory of Bio-pesticide and Chemistry Biology, Ministry of Education, Fujian Agricultural and Forestry University, China) for providing pEarleyGate201, pEarleyGate202, and pEarleyGate203. We also want to thank Mark D. Curtis (University of Zurich) for kindly providing Gateway-destination vectors and S.P. Dinesh-Kumar (Yale University) for providing pTRV1 and pTRV2. Furthermore, this work was supported by grants from the National Natural Science Foundation of China (31501767, 31572136, and 31372061), the Natural Science Foundation of Fujian Province, China (2017J01436), and Young and Middle-aged Teachers Education Scientific Research Project of Fujian Provincial Department of Education (2016). The funders had no role in study design, data collection, data analysis, the decision to publish, or the preparation of the manuscript.

Author Contributions: Zhiqin Liu, Lanping Shi, Sheng Yang, and Shuilin He conceived and designed the experiments. Zhiqin Liu, Lanping Shi, Sheng Yang, Youquan Lin, Yahong Weng, and Xia Li performed the experiments. Zhiqin Liu, Lanping Shi, Sheng Yang, Ansar Hussain, Ali Noman, and Shuilin He analyzed the data. Zhiqin Liu and Shuilin He wrote the paper. Zhiqin Liu and Shuilin He provided guidance on the whole study.

Conflicts of Interest: The authors declare no conflict of interest.

\section{References}

1. Jones, J.D.; Dangl, J.L. The plant immune system. Nature 2006, 444, 323-329. [CrossRef] [PubMed]

2. Hein, I.; Gilroy, E.M.; Armstrong, M.R.; Birch, P.R. The zig-zag-zig in oomycete-plant interactions. Mol. Plant Pathol. 2009, 10, 547-562. [CrossRef] [PubMed]

3. Tsuda, K.; Katagiri, F. Comparing signaling mechanisms engaged in pattern-triggered and effector-triggered immunity. Curr. Opin. Plant Biol. 2010, 13, 459-465. [CrossRef] [PubMed]

4. Thomma, B.P.; Nurnberger, T.; Joosten, M.H. Of pamps and effectors: The blurred pti-eti dichotomy. Plant Cell 2011, 23, 4-15. [CrossRef] [PubMed]

5. Greeff, C.; Roux, M.; Mundy, J.; Petersen, M. Receptor-like kinase complexes in plant innate immunity. Front. Plant Sci. 2012, 3, 209. [CrossRef] [PubMed]

6. Nakahara, K.S.; Masuta, C. Interaction between viral RNA silencing suppressors and host factors in plant immunity. Curr. Opin. Plant Biol. 2014, 20, 88-95. [CrossRef] [PubMed]

7. Lee, M.F.; Lin, S.J.; Wang, N.M.; Wu, H.J.; Chen, Y.H. Plant chitinase III Ziz m 1 stimulates multiple cytokines, most predominantly interleukin-13, from peripheral blood mononuclear cells of latex-fruit allergic patients. Ann. Allergy Asthma Immunol. 2012, 108, 113-116. [CrossRef] [PubMed]

8. Ahmed, N.U.; Park, J.I.; Jung, H.J.; Kang, K.K.; Hur, Y.; Lim, Y.P.; Nou, I.S. Molecular characterization of stress resistance-related chitinase genes of Brassica rapa. Plant Physiol. Biochem. 2012, 58, 106-115. [CrossRef] [PubMed] 
9. Itoh, Y.; Takahashi, K.; Takizawa, H.; Nikaidou, N.; Tanaka, H.; Nishihashi, H.; Watanabe, T.; Nishizawa, Y. Family 19 chitinase of Streptomyces griseus HUT6037 increases plant resistance to the fungal disease. Biosci. Biotechnol. Biochem. 2003, 67, 847-855. [CrossRef] [PubMed]

10. Amian, A.A.; Papenbrock, J.; Jacobsen, H.J.; Hassan, F. Enhancing transgenic pea (Pisum Sativum L.) resistance against fungal diseases through stacking of two antifungal genes (Chitinase and Glucanase). GM Crops 2011, 2, 104-109. [CrossRef] [PubMed]

11. Kalinina, O.; Zeller, S.L.; Schmid, B. Competitive performance of transgenic wheat resistant to powdery mildew. PLoS ONE 2011, 6, e28091. [CrossRef] [PubMed]

12. van Loon, L.C.; Rep, M.; Pieterse, C.M. Significance of inducible defense-related proteins in infected plants. Annu. Rev. Phytopathol. 2006, 44, 135-162. [CrossRef] [PubMed]

13. Gao, Y.; Zan, X.L.; Wu, X.F.; Yao, L.; Chen, Y.L.; Jia, S.W.; Zhao, K.J. Identification of fungus-responsive cis-acting element in the promoter of Brassica juncea chitinase gene, BJCHI1. Plant Sci. 2014, 215-216, 190-198. [CrossRef] [PubMed]

14. Hong, J.K.; Hwang, B.K. Promoter activation of pepper class ii basic chitinase gene, CAChi2, and enhanced bacterial disease resistance and osmotic stress tolerance in the CAChi2-overexpressing Arabidopsis. Planta 2006, 223, 433-448. [CrossRef] [PubMed]

15. Lu, X.; Jiang, W.; Zhang, L.; Zhang, F.; Shen, Q.; Wang, G.; Tang, K. AaERF1 positively regulates the resistance to Botrytis cinerea in Artemisia annua. PLoS ONE 2013, 8, e57657. [CrossRef] [PubMed]

16. Jung, H.W.; Hwang, B.K. Isolation, partial sequencing, and expression of pathogenesis-related cDNA genes from pepper leaves infected by Xanthomonas campestris pv. Vesicatoria. Mol. Plant Microbe Interact. 2000, 13, 136-142. [CrossRef] [PubMed]

17. Hong, J.K.; Jung, H.W.; Kim, Y.J.; Hwang, B.K. Pepper gene encoding a basic class II chitinase is inducible by pathogen and ethephon. Plant Sci. 2000, 159, 39-49. [CrossRef]

18. Hong, J.K.; Hwang, B.K. Induction by pathogen, salt and drought of a basic class II chitinase mRNA and its in situ localization in pepper (Capsicum annuum). Physiol. Plant 2002, 114, 549-558. [CrossRef] [PubMed]

19. Kim, D.S.; Kim, N.H.; Hwang, B.K. The Capsicum annuum class IV chitinase chitiv interacts with receptor-like cytoplasmic protein kinase PIK1 to accelerate PIK1-triggered cell death and defence responses. J. Exp. Bot. 2015, 66, 1987-1999. [CrossRef] [PubMed]

20. Lee, S.Y.; Tindwa, H.; Lee, Y.S.; Naing, K.W.; Hong, S.H.; Nam, Y.; Kim, K.Y. Biocontrol of anthracnose in pepper using chitinase, $\beta-1,3$ glucanase, and 2-furancarboxaldehyde produced by streptomyces cavourensis SY224. J. Microbiol. Biotechnol. 2012, 22, 1359-1366. [CrossRef] [PubMed]

21. Lee, I.A.; Low, D.; Kamba, A.; Llado, V.; Mizoguchi, E. Oral caffeine administration ameliorates acute colitis by suppressing chitinase 3-like 1 expression in intestinal epithelial cells. J. Gastroenterol. 2014, 49, 1206-1216. [PubMed]

22. Liu, Z.Q.; Qiu, A.L.; Shi, L.P.; Cai, J.S.; Huang, X.Y.; Yang, S.; Wang, B.; Shen, L.; Huang, M.K.; Mou, S.L.; et al. SRC2-1 is required in PCINF1-induced pepper immunity by acting as an interacting partner of PcINF1. J. Exp. Bot. 2015, 66, 3683-3698. [PubMed]

23. Liu, Y.; Schiff, M.; Dinesh-Kumar, S.P. Virus-induced gene silencing in tomato. Plant J. 2002, 31, 777-786. [PubMed]

24. Choi, H.W.; Kim, N.H.; Lee, Y.K.; Hwang, B.K. The pepper extracellular xyloglucan-specific endo- $\beta$-1,4-glucanase inhibitor protein gene, CaXEGIP1, is required for plant cell death and defense responses. Plant Physiol. 2013, 161, 384-396. [PubMed]

25. Choi, D.S.; Hwang, I.S.; Hwang, B.K. Requirement of the cytosolic interaction between PATHOGENESIS-RELATED PROTEIN10 and LEUCINE-RICH REPEAT PROTEIN1 for cell death and defense signaling in pepper. Plant Cell 2012, 24, 1675-1690. [PubMed]

26. Knoth, C.; Eulgem, T. The oomycete response gene LURP1 is required for defense against Hyaloperonospora parasitica in Arabidopsis thaliana. Plant J. 2008, 55, 53-64. [PubMed]

27. Oliveira, M.B.; Junior, M.L.; Grossi-de-Sa, M.F.; Petrofeza, S. Exogenous application of methyl jasmonate induces a defense response and resistance against Sclerotinia sclerotiorum in dry bean plants. J. Plant Physiol. 2015, 182, 13-22. [PubMed]

28. Widjaja, I.; Lassowskat, I.; Bethke, G.; Eschen-Lippold, L.; Long, H.H.; Naumann, K.; Dangl, J.L.; Scheel, D.; Lee, J. A protein phosphatase 2C, responsive to the bacterial effector AvrRpm1 but not to the AvrB effector, regulates defense responses in Arabidopsis. Plant J. 2010, 61, 249-258. [PubMed] 
29. Buscaill, P.; Rivas, S. Transcriptional control of plant defence responses. Curr. Opin. Plant. Biol. 2014, 20, 35-46. [PubMed]

30. Moore, J.W.; Loake, G.J.; Spoel, S.H. Transcription dynamics in plant immunity. Plant Cell 2011, 23, $2809-2820$. [PubMed]

31. Adachi, H.; Nakano, T.; Miyagawa, N.; Ishihama, N.; Yoshioka, M.; Katou, Y.; Yaeno, T.; Shirasu, K.; Yoshioka, H. WRKY transcription factors phosphorylated by MAPK regulate a plant immune NADPH oxidase in Nicotiana benthamiana. Plant Cell 2015, 27, 2645-2663. [CrossRef] [PubMed]

32. Eulgem, T.; Weigman, V.J.; Chang, H.S.; McDowell, J.M.; Holub, E.B.; Glazebrook, J.; Zhu, T.; Dangl, J.L. Gene expression signatures from three genetically separable resistance gene signaling pathways for downy mildew resistance. Plant Physiol. 2004, 135, 1129-1144. [CrossRef] [PubMed]

33. Curto, M.; Krajinski, F.; Schlereth, A.; Rubiales, D. Transcriptional profiling of Medicago truncatula during Erysiphe pisi infection. Front. Plant Sci. 2015, 6, 517. [CrossRef] [PubMed]

34. Eulgem, T.; Rushton, P.J.; Robatzek, S.; Somssich, I.E. The WRKY superfamily of plant transcription factors. Trends Plant Sci. 2000, 5, 199-206. [CrossRef]

35. Eulgem, T.; Rushton, P.J.; Schmelzer, E.; Hahlbrock, K.; Somssich, I.E. Early nuclear events in plant defence signalling: Rapid gene activation by WRKY transcription factors. EMBO J. 1999, 18, 4689-4699. [CrossRef] [PubMed]

36. Ulker, B.; Somssich, I.E. WRKY transcription factors: From DNA binding towards biological function. Curr. Opin. Plant Biol. 2004, 7, 491-498. [CrossRef] [PubMed]

37. Zander, M.; La Camera, S.; Lamotte, O.; Metraux, J.P.; Gatz, C. Arabidopsis thaliana class-II TGA transcription factors are essential activators of jasmonic acid/ethylene-induced defense responses. Plant J. 2010, 61, 200-210. [CrossRef] [PubMed]

38. Fink, J.S.; Verhave, M.; Kasper, S.; Tsukada, T.; Mandel, G.; Goodman, R.H. The CGTCA sequence motif is essential for biological activity of the vasoactive intestinal peptide gene cAMP-regulated enhancer. Proc. Natl. Acad. Sci. USA 1988, 85, 6662-6666. [CrossRef] [PubMed]

39. Ramonell, K.; Berrocal-Lobo, M.; Koh, S.; Wan, J.; Edwards, H.; Stacey, G.; Somerville, S. Loss-of-function mutations in chitin responsive genes show increased susceptibility to the powdery mildew pathogen Erysiphe cichoracearum. Plant Physiol. 2005, 138, 1027-1036. [CrossRef] [PubMed]

40. Rowland, O.; Ludwig, A.A.; Merrick, C.J.; Baillieul, F.; Tracy, F.E.; Durrant, W.E.; Fritz-Laylin, L.; Nekrasov, V.; Sjolander, K.; Yoshioka, H.; et al. Functional analysis of AVR9/CF-9 rapidly elicited genes identifies a protein kinase, ACIK1, that is essential for full Cf-9-dependent disease resistance in tomato. Plant Cell 2005, 17, 295-310. [CrossRef] [PubMed]

41. Bartsch, M.; Gobbato, E.; Bednarek, P.; Debey, S.; Schultze, J.L.; Bautor, J.; Parker, J.E. Salicylic acid-independent ENHANCED DISEASE SUSCEPTIBILITY1 signaling in Arabidopsis immunity and cell death is regulated by the monooxygenase FMO1 and the nudix hydrolase Nudt7. Plant Cell 2006, 18, 1038-1051. [CrossRef] [PubMed]

42. Veronese, P.; Nakagami, H.; Bluhm, B.; Abuqamar, S.; Chen, X.; Salmeron, J.; Dietrich, R.A.; Hirt, H.; Mengiste, T. The membrane-anchored BOTRYTIS-INDUCED KINASE1 plays distinct roles in Arabidopsis resistance to necrotrophic and biotrophic pathogens. Plant Cell 2006, 18, 257-273. [CrossRef] [PubMed]

43. Pandey, S.P.; Somssich, I.E. The role of WRKY transcription factors in plant immunity. Plant Physiol. 2009, 150, 1648-1655. [CrossRef] [PubMed]

44. Ishihama, N.; Yoshioka, H. Post-translational regulation of WRKY transcription factors in plant immunity. Curr. Opin. Plant Biol. 2012, 15, 431-437. [CrossRef] [PubMed]

45. Bhattacharjee, S.; Garner, C.M.; Gassmann, W. New clues in the nucleus: Transcriptional reprogramming in effector-triggered immunity. Front. Plant Sci. 2013, 4, 364. [CrossRef] [PubMed]

46. Stewart, C.N., Jr.; Via, L.E. A rapid CTAB DNA isolation technique useful for RAPD fingerprinting and other PCR applications. Biotechniques 1993, 14, 748-750. [PubMed]

47. Higo, K.; Ugawa, Y.; Iwamoto, M.; Korenaga, T. Plant cis-acting regulatory DNA elements (PLACE) database: 1999. Nucleic Acids Res. 1999, 27, 297-300. [CrossRef] [PubMed]

48. Lescot, M.; Dehais, P.; Thijs, G.; Marchal, K.; Moreau, Y.; Van de Peer, Y.; Rouze, P.; Rombauts, S. PlantCARE, a database of plant cis-acting regulatory elements and a portal to tools for in silico analysis of promoter sequences. Nucleic Acids Res. 2002, 30, 325-327. [CrossRef] [PubMed] 
49. Earley, K.W.; Haag, J.R.; Pontes, O.; Opper, K.; Juehne, T.; Song, K.; Pikaard, C.S. Gateway-compatible vectors for plant functional genomics and proteomics. Plant J. 2006, 45, 616-629. [CrossRef] [PubMed]

50. Muller, A.J.; Mendel, R.R.; Schiemann, J.; Simoens, C.; Inze, D. High meiotic stability of a foreign gene introduced into tobacco by Agrobacterium-mediated transformation. Mol. Gen. Genet. 1987, 207, 171-175. [CrossRef] [PubMed]

51. Wirth, S.J.; Wolf, G.A. Dye-labelled substrates for the assay and detection of chitinase and lysozyme activity. J. Microbiol. Meth. 1990, 12, 197-205. [CrossRef]

52. Hsu, S.C.; Lockwood, J.L. Powdered chitin agar as a selective medium for enumeration of actinomycetes in water and soil. Appl. Microbiol. 1975, 29, 422-426. [PubMed]

53. Miller, G.L. Use of dinitrosalicylic acid reagent for determination of reducing sugar. Anal. Chem. 1959, 31, 426-428. [CrossRef]

54. Li, P.; Chen, L.; Zhou, Y.; Xia, X.; Shi, K.; Chen, Z.; Yu, J. Brassinosteroids-induced systemic stress tolerance was associated with increased transcripts of several defence-related genes in the phloem in Cucumis sativus. PLoS ONE 2013, 8, e66582. [CrossRef] [PubMed]

55. Cai, H.; Yang, S.; Yan, Y.; Xiao, Z.; Cheng, J.; Wu, J.; Qiu, A.; Lai, Y.; Mou, S.; Guan, D.; et al. CaWRKY6 transcriptionally activates CaWRKY40, regulates Ralstonia solanacearum resistance, and confers high-temperature and high-humidity tolerance in pepper. J. Exp. Bot. 2015, 66, 3163-3174. [CrossRef] [PubMed]

56. Dang, F.; Wang, Y.; She, J.; Lei, Y.; Liu, Z.; Eulgem, T.; Lai, Y.; Lin, J.; Yu, L.; Lei, D.; et al. Overexpression of CaWRKY27, a subgroup IIe WRKY transcription factor of Capsicum annuum, positively regulates tobacco resistance to Ralstonia solanacearum infection. Physiol. Plant 2014, 150, 397-411. [CrossRef] [PubMed]

57. Zhang, Q.; Kuang, H.; Chen, C.; Yan, J.; Do-Umehara, H.C.; Liu, X.Y.; Dada, L.; Ridge, K.M.; Chandel, N.S.; Liu, J. The kinase Jnk2 promotes stress-induced mitophagy by targeting the small mitochondrial form of the tumor suppressor ARF for degradation. Nat. Immunol. 2015, 16, 458-466. [CrossRef] [PubMed]

58. Livak, K.J.; Schmittgen, T.D. Analysis of relative gene expression data using real-time quantitative PCR and the $2^{-\Delta \Delta C t}$ method. Methods 2001, 25, 402-408. [CrossRef] [PubMed] 